\title{
Review Article \\ Roles of Akt and SGK1 in the Regulation of Renal Tubular Transport
}

\author{
Nobuhiko Satoh, ${ }^{1}$ Motonobu Nakamura, ${ }^{1}$ Masashi Suzuki, ${ }^{1}$ Atsushi Suzuki, ${ }^{1}$ \\ George Seki, ${ }^{2}$ and Shoko Horita ${ }^{1}$ \\ ${ }^{1}$ Department of Nephrology, The University of Tokyo Hospital, 7-3-1 Hongo, Bunkyo, Tokyo 113-8655, Japan \\ ${ }^{2}$ Yaizu City Hospital, 1000 Dobara, Yaizu, Shizuoka 425-8505, Japan \\ Correspondence should be addressed to Shoko Horita; shorita-tky@umin.ac.jp
}

Received 3 July 2015; Accepted 6 September 2015

Academic Editor: Goutam Ghosh Choudhury

Copyright (C) 2015 Nobuhiko Satoh et al. This is an open access article distributed under the Creative Commons Attribution License, which permits unrestricted use, distribution, and reproduction in any medium, provided the original work is properly cited.

A serine/threonine kinase Akt is a key mediator in various signaling pathways including regulation of renal tubular transport. In proximal tubules, Akt mediates insulin signaling via insulin receptor substrate 2 (IRS2) and stimulates sodium-bicarbonate cotransporter (NBCe1), resulting in increased sodium reabsorption. In insulin resistance, the IRS2 in kidney cortex is exceptionally preserved and may mediate the stimulatory effect of insulin on NBCel to cause hypertension in diabetes via sodium retention. Likewise, in distal convoluted tubules and cortical collecting ducts, insulin-induced Akt phosphorylation mediates several hormonal signals to enhance sodium-chloride cotransporter (NCC) and epithelial sodium channel (ENaC) activities, resulting in increased sodium reabsorption. Serum- and glucocorticoid-inducible kinase 1 (SGK1) mediates aldosterone signaling. Insulin can stimulate SGK1 to exert various effects on renal transporters. In renal cortical collecting ducts, SGK1 regulates the expression level of ENaC through inhibition of its degradation. In addition, SGK1 and Akt cooperatively regulate potassium secretion by renal outer medullary potassium channel (ROMK). Moreover, sodium-proton exchanger 3 (NHE3) in proximal tubules is possibly activated by SGK1. This review focuses on recent advances in understanding of the roles of Akt and SGK1 in the regulation of renal tubular transport.

\section{Introduction}

Renal tubules reabsorb most of water and electrolytes such as sodium, bicarbonate, and phosphate from glomerular filtrate. In particular, sodium reabsorption is regulated by various transporters along the nephron and to elucidate the mechanism of its regulation is important because excess renal sodium retention causes hypertension in metabolic syndrome that leads to cardiovascular disease [1]. Several hormonal signaling pathways have been shown to be involved in the expression and activation of such renal tubular transporters.

Akt is a serine/threonine kinase that was initially identified as an oncogene, as suggested by its name (formed from AK mouse + transforming or thymoma) [2]. It is also known as protein kinase $\mathrm{B}(\mathrm{PKB})$. Insulin stimulates phosphorylation of Akt and activated Akt exerts various biological effects such as cell growth and survival, angiogenesis, and metabolism by regulating downstream effectors [3]. For example, Akt induces the translocation of glucose transporter 4 (GLUT4) from intracellular sites to the plasma membrane, triggering glucose uptake into muscle and adipocytes. The insulin-induced Akt activation also impacts renal tubular transport through several signaling pathways and thus regulates sodium reabsorption.

SGK1 was initially discovered as a serum- and glucocorticoid-inducible serine/threonine kinase in rat mammary tumor cells $[4,5]$. It is ubiquitously expressed in most tissues under the control of hormones such as glucocorticoids and mineral corticoids and regulated at the transcriptional level by osmotic changes [6]. Similar to Akt, SGK1 is also known to be a powerful regulator of metabolism, transcription, enzyme activity, and renal transport [7]. Furthermore, insulin also has stimulatory effects on SGK1 and activated SGK1 acts upon renal tubular transport independently or sometimes cooperatively with Akt [8]. In particular, SGK1 has a key 


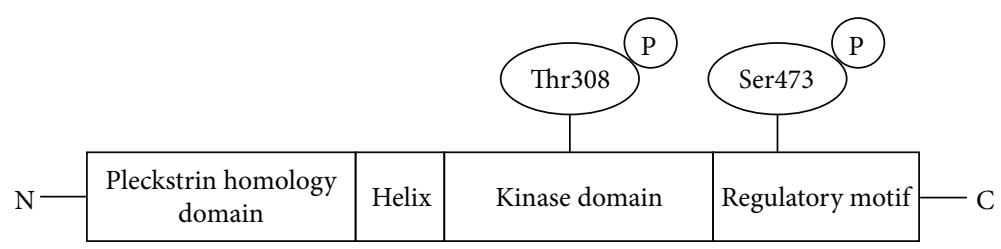

FIGURE 1: Schematic structure of Akt1. Akt1 has 480 amino acids and it is composed of four domains: the pleckstrin homology domain, the helix domain, the kinase domain, and the regulatory domain. The kinase and the regulatory domains have phosphorylation sites at Thr308 and Ser473, respectively. Phosphorylation of these sites induces Aktl activation.

role in $\mathrm{NaCl}$ homeostasis through regulating the epithelial sodium channel (ENaC) [9] in cortical collecting ducts (CCDs). Other than that, it stimulates a large number of renal tubular transporters, maintaining electrolyte balance.

In this review, we focus on the role of Akt- and SGK1mediated signaling pathways in the regulation of renal tubular transport, especially sodium homeostasis, with emphasis on recent advances.

\section{Akt Signaling}

2.1. Akt: Isoforms, Distribution, and Structures. Akt has three isoforms, Akt1, Akt2, and Akt3. Akt1 is widely expressed in most tissues and has been implicated in cell growth and survival. In contrast, the expression patterns and functions of Akt 2 and Akt3 are more limited. Akt2 is highly expressed in insulin-sensitive tissues such as skeletal muscle and adipocytes, where it mediates insulin-induced glucose uptake [10]. Akt3 is predominantly expressed in brains and testes, where it may be involved in pathophysiology of neurological disorders [11]. Akt1, Akt2, and Akt3 are encoded by distinct loci located on 14q32.3, 19q13.2, and 1q43-q44, respectively. All of the Akts have similar structures that include a pleckstrin homology $(\mathrm{PH})$ domain, a helix region, a kinase domain, and a regulatory motif as shown in Figure 1 [11]. Human Akt1 has key phosphorylation sites, Thr308 and Ser473 in the kinase domain and the regulatory motif, respectively. Similarly, Akt2 and Akt3 also have phosphorylation sites at homologous positions.

2.2. Akt in Insulin Signaling Pathway. Among three Akt isoforms, Akt2 is essential for insulin-mediated glucose uptake into muscle and adipocytes. Binding of insulin to the insulin receptor (IR) first activates intrinsic receptor kinase function, resulting in phosphorylation of several key sites on the intracellular domain of the receptor. Insulin receptor substrate (IRS) binds to these phosphorylated sites on IR and in turn is activated via phosphorylation. Activated IRS protein binds to phosphatidylinositol 3-kinase (PI3K) that converts phosphatidylinositol $(4,5)$-bisphosphate (PIP2) to phosphatidylinositol $(1,4,5)$-triphosphate (PIP3). PIP3 facilitates translocation of phosphoinositide-dependent protein kinase 1 (PDK1) and Akt2 close to the membrane for activation of PDK1 and Akt2. In addition, activated PDK1 phosphorylates Akt2 at Thr309. On the other hand, PI3K also activates another mediator, the mammalian target of rapamycin complex 2 (mTORC2) which phosphorylates Akt2 at Ser474 [10].
Eventually, activated Akt2 induces the translocation of GLUT4 from intracellular sites to the plasma membrane, triggering glucose uptake into muscle and adipocytes. The insulin/Akt signaling cascade including Akt2-mediated GLUT4 translocation is summarized in Figure 2.

2.3. Akt and Insulin-Induced GLUT4 Translocation. GLUT4 is a member of the facilitative glucose transporter family that is predominantly expressed in muscle and fat tissues [12]. GLUT4 may also be expressed in renal tubules [13]; however, its detailed distribution in the kidney has not yet been clarified. Although there is a dispute regarding the role of Akt 2 in the translocation of GLUT4 to the plasma membrane, Gonzalez and McGraw showed that Akt2 is indispensable for the insulin-stimulated initiation of GLUT4 translocation. By pharmacological inhibition or knockdown with short hairpin RNA, they showed that the Akt substrate AS160 is a key factor in GLUT4 translocation [14].

2.4. Akt and Tubular Transport. Proximal tubules (PTs) reabsorb most of water, nutrients, and electrolytes such as glucose, sodium, bicarbonate, and phosphate from glomerular filtrate. In the PTs, sodium is reabsorbed primarily by sodium-proton exchanger 3 (NHE3) on the apical side and by sodiumbicarbonate cotransporter (NBCel) on the basolateral side. Several hormones and mediators such as angiotensin II, nitric oxide, dopamine, and insulin are known to regulate these transporters. $[1,15]$. Among them, insulin has previously been shown to stimulate PT sodium and volume transport $[16,17]$.

By using IRS1 ${ }^{-/-}$and IRS2 $2^{-/-}$mice, we previously showed that the IRS2/PI3K pathway plays a major role in the stimulation of renal proximal absorption by insulin [18]. Additionally, by gene silencing in rats with short interfering RNA (siRNA) against IRS1 or IRS2, we also showed that while IRS2 mediates the stimulatory effect of insulin on PT transport, IRS1 mediates the stimulatory effect of insulin on glucose uptake into adipocytes [19].

Interestingly, we also found that while the stimulatory effect of insulin on glucose uptake and Akt phosphorylation in adipocytes were markedly reduced, the insulin-induced stimulation of NBCel and Akt phosphorylation in kidney cortex were completely preserved in insulin resistant human patients and Otsuka Long-Evans Tokushima Fatty (OLETF) rats [19]. In insulin resistance, the IRS2 in kidney cortex is exceptionally preserved and may mediate the stimulatory effect of insulin on NBCel. 


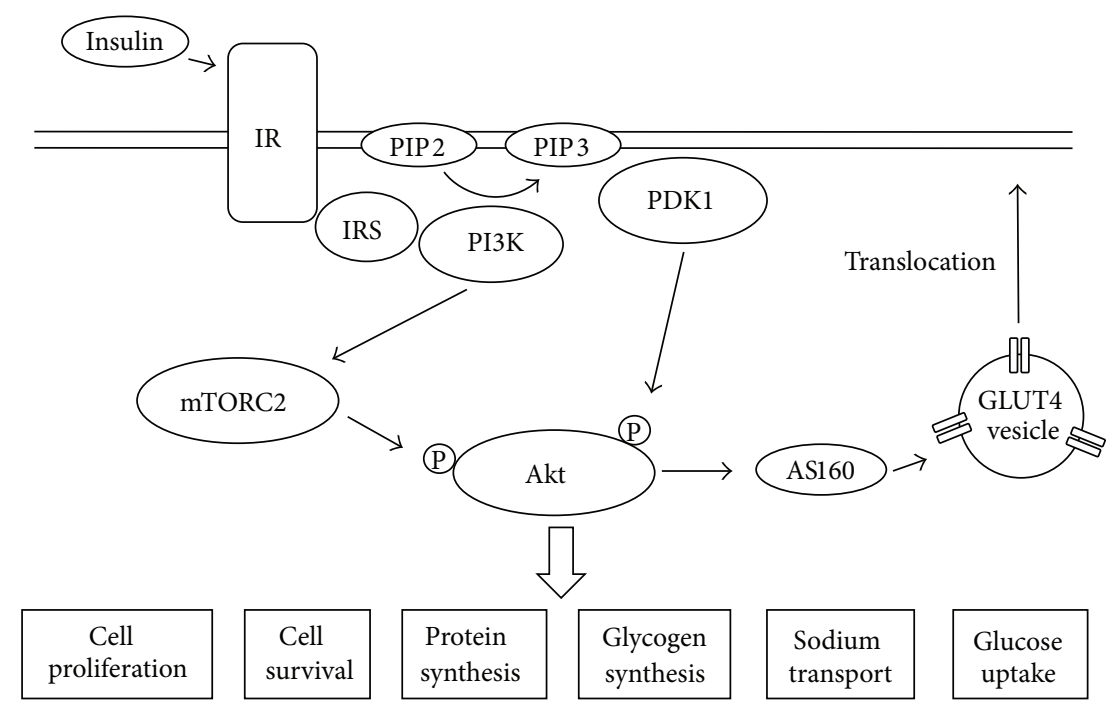

FIGURE 2: Insulin-mediated Akt signaling. Insulin binding to insulin receptor induces Akt activation through IRS/PI3K pathway. mTORC2 also activates Akt. Activated Akt regulates various biological functions such as cell survival and proliferation, protein synthesis, glycogen synthesis, sodium transport, and glucose uptake through translocation of GLUT4. IR: insulin receptor, IRS: insulin receptor substrate, PI3K: phosphatidylinositol 3-kinase, PDK1: phosphoinositide-dependent protein kinase 1, and mTORC2: mammalian target of rapamycin complex 2 .

These findings strongly suggest that hyperinsulinemia in insulin resistance induces volume expansion and hypertension via PT transport stimulation and Akt mediates the stimulatory effect of insulin on NBCel as a downstream effector of insulin/IRS2/PI3K pathway. Akt2 is reported to be involved in the regulation of sodium-coupled glucose transport [20] and sodium-coupled phosphate transport [21]. SGK1 may be also involved in the regulation of PT transport as discussed below. Thus, further investigation would be required to elucidate how Akt actually mediates the insulin action on PT transport. The effect of insulin on PT is summarized in Figure 3.

2.5. Akt, With-No-Lysine Kinases (WNKs), and Sodium-Chloride Cotransporter (NCC). Sodium-chloride cotransporter (NCC) is primarily expressed in distal convoluted tubules and mediates sodium and chloride reabsorption [22]. It is under the control of several hormones such as insulin, angiotensin II, glucocorticoids, and aldosterone [22]. Among these hormones, aldosterone has previously been recognized to stimulate NCC activity but the detailed mechanism of this process is only now being clarified. The discovery of with-no-lysine kinases (WNKs) [23] may provide a clue for deciphering this complicated mechanism.

WNK has several isoforms and variants including WNK1 (chromosome 12p13.3), WNK2 (chromosome 9q22.31), WNK3 (chromosome Xp11.22), WNK4 (chromosome 17q21.31), and kidney-specific WNK1 (KS-WNK1). In contrast to the other isoforms, the significance of WNK2 has not been established [24]. The physiological and pathological roles of WNKs are described in excellent reviews, for example, those by Hoorn and Ellison and by Uchida $[25,26]$.

In distal convoluted tubule (DCT) cells, WNKs play crucial roles in the regulation of NCC. Along with phosphorylating STE20/SPS1-related proline/alanine-rich kinase

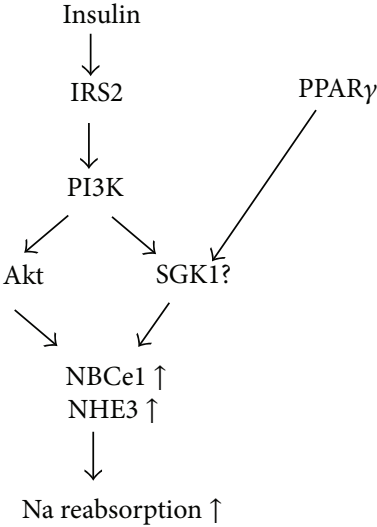

FIGURE 3: Effects of insulin on PT sodium transport. Insulin stimulates NBCe1 and NHE3 via IRS2/PI3K/Akt pathway. PPAR $\gamma$ and SGK1 may be involved in the stimulatory effect on PT sodium transport. NBCel: sodium-bicarbonate cotransporter 1, NHE3: sodiumproton exchanger 3, PPAR $\gamma$ : peroxisome proliferator-activated receptor gamma, and SGK1: serum- and glucocorticoid-inducible kinase 1.

(SPAK) and oxidative-stress-responsive kinase 1 (OSR1), WNK3 induces NCC translocation from cytosol to luminal membrane. Phosphorylated OSR1/SPAK then activates NCC by phosphorylation (WNK-OSR1/SPAK-NCC phosphorylation cascade). In contrast, WNK4 had been shown to inhibit NCC by promoting lysosomal degradation [22, 25]. However, Takahashi and colleagues recently showed that insulin fails to stimulate NCC in $\mathrm{WNK}^{-/-}$mice [27]. Moreover, these authors found that transgenic mice overexpressing WNK4 exhibit pseudohypoaldosteronism type II (PHA-II) [28], suggesting that WNK4 positively regulates NCC. Further 


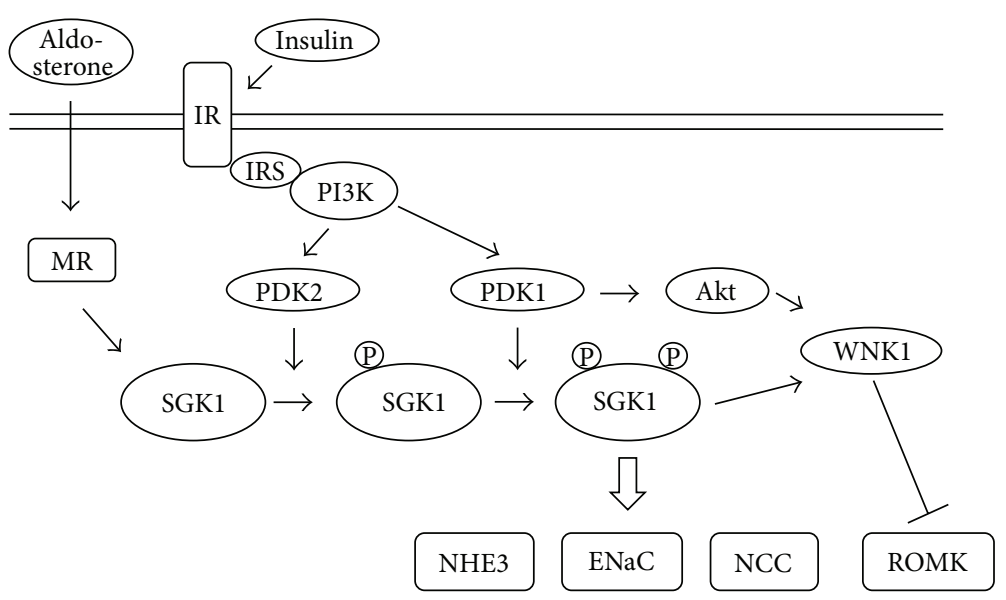

FIGURE 4: SGK1-mediated signaling pathway. Aldosterone stimulates SGK1 via mineralocorticoid receptor (MR), while insulin stimulates SGK1 via IRS/PI3K pathway. SGK1 is phosphorylated twofold by PDK1 and PDK2. Activated SGK1 exerts stimulatory effects on sodium transport through ENaC, NCC, and NHE3. Also, SGK1 regulates potassium secretion through ROMK. SGK1: serum- and glucocorticoidinducible kinase 1, IRS: insulin receptor substrate, PI3K: phosphatidylinositol 3-kinase, PDK1: phosphoinositide-dependent protein kinase 1, WNK1: with-no-lysine kinase 1, ENaC: epithelial $\mathrm{Na}^{+}$channel, ROMK: renal outer medullary potassium channel, and NHE3: sodium-proton exchanger 3 .

experiments would be required to establish the precise role of WNK4 in the regulation of NCC.

Recently, Akt has been suggested to mediate insulininduced WNK-OSR1/SPAK-NCC phosphorylation cascade. In $d b / d b$ diabetic mice that show hyperinsulinemia and high thiazide sensitivity, the phosphorylation of renal Akt as well as NCC is increased. Furthermore, this increased phosphorylation of NCC is corrected by specific inhibitors for PI3K or Akt, suggesting that the insulin/PI3K/Akt pathway regulates WNK-OSR1/SPAK-NCC phosphorylation cascade [29]. Insulin also induces strong renal Akt phosphorylation in another model of metabolic syndrome Zucker obese rats, resulting in NCC phosphorylation [30]. The stimulation of NCC may be also involved in hyperinsulinemia-induced hypertension observed in metabolic syndrome.

\section{SGK Signaling}

3.1. SGK: Isoforms, Distribution, and Structures. To date, SGK1 is known to have two isoforms, SGK2 and SGK3. Like SGK1, SGK3 is ubiquitously expressed in a variety of tissues; however, the expression of SGK2 is limited to liver, kidney, pancreas, and brain. In human, SGK1, SGK2, and SGK3 are encoded by different loci, 6q23, 20q12, and 8q12.3, respectively. About $80 \%$ of amino acids in catalytic domain of SGK2 and SGK3 are identical to that of SGK1 [31].

3.2. SGK1 in Insulin Signaling Pathway. Insulin can stimulate SGK1 through insulin/IRS/PI3K pathway. In the insulin/PI3K pathway, SGK1 is phosphorylated twofold by PDK1 and PDK2. Thus, activated SGK1 exerts a variety of effects on renal transporters, including the stimulation of ENaC, NCC, and NHE3 and the inhibition of renal outer medullary potassium channel (ROMK) through phosphorylation of WNK1, as will be discussed below $[8,31,32]$. Figure 4 summarizes the typical signal transduction of SGK1.

3.3. SGK1 and Tubular Transport. SGK1 is well known to be an efficient regulator of $\mathrm{ENaC}$ [9] in CCD. It activates $\mathrm{ENaC}$ through several pathways [33] and among these pathways, inhibition of E3 ubiquitin ligase, neural precursor cell-expressed, developmentally downregulated protein 4-2 (Nedd4-2) seems to be the most important.

Nedd4-2 binds to and ubiquitinates $\mathrm{ENaC}$ at the cell surface, triggering its internalization and degradation [34]. SGK1 phosphorylates and inhibits Nedd4-2, resulting in increased expression level of ENaC. In fact, kidney tubulespecific SGK1 knockout mice [35] exhibit decreased phosphorylation of Nedd4-2 and decreased expression of all ENaC subunits. Moreover, expression of NCC in the kidney was decreased. These findings suggest that NCC is also regulated by SGK1 [36, 37]. Ronzaud and colleagues showed that nephron-specific Nedd4-2-deficient mice exhibit saltdependent hypertension due to the upregulation of NCC [38]. Therefore, Nedd4-2 may be involved in inhibition of both $\mathrm{ENaC}$ and NCC. On the other hand, WNK4, which is phosphorylated by SGK1 at serine 1169, is also involved in the regulation of ENaC. [39].

Recently, SGK1 is also suggested to modulate PT transport. For example, SGK1 has been shown to be upregulated by angiotensin II, which results in the activation of NHE3 and increased PT sodium reabsorption [32, 40, 41]. Fuster and colleagues also showed that SGK1 mediates the chronic stimulatory effect of insulin on NHE3 in a cell line model of PT [17]. Moreover, SGK1 might mediate NHE3 activation via peroxisome proliferator-activated receptor gamma (PPAR $\gamma)$ in human PT cells [40]. Another transporter in PT, sodiumdicarboxylate cotransporter, is also upregulated by both SGK1 and Akt [42]. 


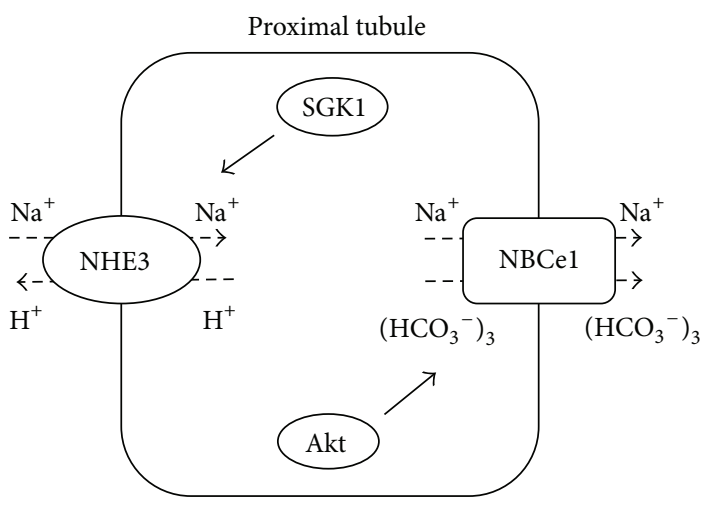

(a)

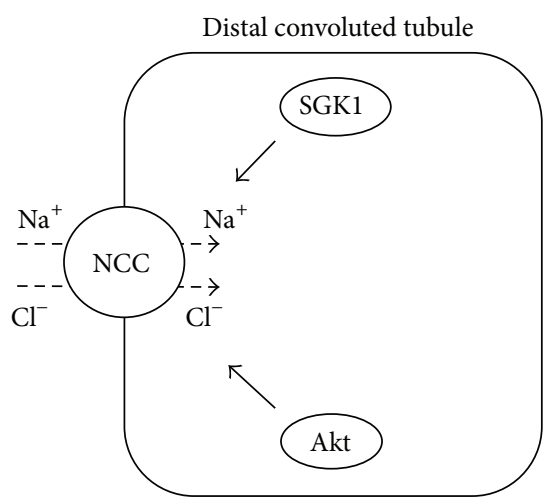

(b)

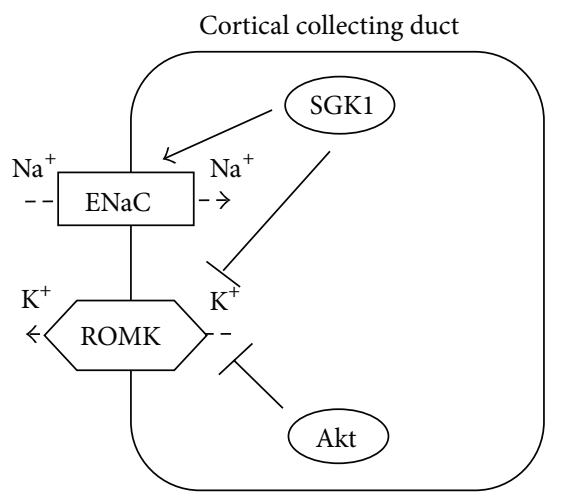

(c)

FIGURE 5: Roles of Akt and SGK1 in transport regulation at each nephron segment: Akt and SGK1 modify renal tubular transport by activating or inhibiting several transporters at each nephron segment. Targets and functions of the kinases in proximal tubules, distal convoluted tubules, and cortical collecting ducts are shown in (a), (b), and (c), respectively.

\section{Cross-Talk between Akt and SGK1 in the Regulation of ROMK}

ROMK, also called Kir1.1 [43, 44], regulates potassium secretion in the outer medullary collecting duct, thereby playing an important role in the homeostasis of potassium. Interestingly, both Aktl and SGK1 regulate the expression of ROMK. Cheng and Huang [8] found that PI3K-activating ligands like insulin or IGF-1 inhibited ROMK by facilitating its endocytosis via phosphorylation of Akt, SGK1, and WNK1. Knockdown of WNK1 prevented the inhibitory effect of insulin on ROMK, and phosphorylation at Thr58 of WNK1 was indispensable for the insulin effect. The authors concluded that both Akt and SGK1 can cooperatively inhibit ROMK through WNK1 phosphorylation.

\section{Recent Advances in the Clinical Development of Akt and SGK Inhibitors}

Akt, as mentioned above, plays an important role in signaling pathways of cell growth, cell survival, glucose metabolism, and renal tubular transport. In particular, several lines indicate that Akt isoforms are overexpressed or hyperactivated in a broad range of human cancers through dysregulated activation of PI3K/Akt pathway. Furthermore, the activation of
Akt is shown to be related with resistance to chemotherapy or radiotherapy and thus involved in the progression or poor prognosis in some types of tumors $[45,46]$. Therefore, Akt and other components in upstream or downstream of PI3K pathway are expected to be a key target for therapeutic intervention [47].

Several drugs targeting each component in PI3K/Akt pathway are now being investigated in a variety of tumors $[48,49]$. For example, an allosteric Akt inhibitor, MK-2206, has been reported to exert potent inhibitory effect on tumor growth in combination with other cytotoxic agents or receptor tyrosine kinase inhibitors and recently entered human clinical trials. Phase II study of MK-2206 as a single agent has revealed that it has favorable toxicity profile with a mild response in lymphoma and further study for mechanismbased combination therapy would be required [50].

SGK1 also regulates cell growth and survival as a downstream effector of PI3K pathway, and it is overexpressed in some types of tumors including colon cancer, medulloblastoma, prostate cancer, ovarian tumors, and non-small cell lung cancer, but not in all tumors $[51,52]$. A previous study showed that $S g k 1(-/-)$ mice exhibited significantly fewer colonic tumors than $S g k 1(+/+)$ mice after chemical cancerogenesis, suggesting the therapeutic possibility of SGK1 inhibitors [53]. Indeed, a highly selective SGK1 inhibitor, 
EMD638683, has been shown to suppress colonic tumor growth in vivo [54]. Furthermore, GSK650394 that functionally inhibits enzymatic activity of SGK1 attenuated androgenmediated growth of the prostate cancer cell line [55].

Thus, components in PI3K pathway contribute to the development of a variety of cancers and selective use of different inhibitors in the pathway would be expected in cancer therapeutics. On the other hand, the modulators of Akt and SGK1 as the therapeutics for diabetes mellitus and other metabolic diseases have not been developed.

\section{Conclusion}

In this review, we have summarized the roles of Akt and SGK1 in renal tubular transport. As a downstream effector of insulin/IRS/PI3K cascade, Akt stimulates sodium reabsorption in several nephron segments. On the other hand, SGK1, activated by PI3K-mediated signaling pathway, also acts upon renal tubular transport independently or sometimes cooperatively with Akt. Figure 5 summarizes the roles of Akt and SGK1 in transport regulation at each nephron segment.

In PTs, Akt stimulates NBCel to increase sodium reabsorption and SGK1 possibly activates NHE3. In DCTs, $\mathrm{PI} 3 \mathrm{~K} / \mathrm{Akt}$ signaling pathway is thought to activate WNKOSR1/SPAK-NCC phosphorylation cascade to stimulate NCC. It is presumed that SGK1 also mediates NCC activation in part; however, the precise mechanism has not been clarified. In CCDs, SGK1 regulates the expression level of $\mathrm{ENaC}$ through inhibition of its degradation by Nedd4-2. On the other hand, both Akt and SGK1 are involved in insulinmediated ROMK inhibition via phosphorylation of WNK1.

However, how Akt and SGK1 actually regulate the activation of those transporters has not yet been clarified. Furthermore, the precise interaction between Akt and SGK1 in renal tubules also remains to be determined.

\section{Conflict of Interests}

The authors declare that there is no conflict of interests regarding the publication of this paper.

\section{Acknowledgment}

This review was supported in part by the Grant no. 25461241 to Shoko Horita from the Ministry of Education, Culture, Sports, Science and Technology of Japan.

\section{References}

[1] S. Horita, G. Seki, H. Yamada, M. Suzuki, K. Koike, and T. Fujita, "Roles of renal proximal tubule transport in the pathogenesis of hypertension," Current Hypertension Reviews, vol. 9, no. 2, pp. 148-155, 2013.

[2] S. P. Staal, "Molecular cloning of the akt oncogene and its human homologues AKT1 and AKT2: amplification of AKT1 in a primary human gastric adenocarcinoma," Proceedings of the National Academy of Sciences of the United States of America, vol. 84, no. 14, pp. 5034-5037, 1987.
[3] B. D. Manning and L. C. Cantley, "AKT/PKB signaling: navigating downstream," Cell, vol. 129, no. 7, pp. 1261-1274, 2007.

[4] G. L. Firestone, J. R. Giampaolo, and B. A. O’Keeffe, “Stimulusdependent regulation of serum and glucocorticoid inducible protein kinase (SGK) transcription, subcellular localization and enzymatic activity," Cellular Physiology and Biochemistry, vol. 13, no. 1, pp. 1-12, 2003.

[5] M. K. Webster, L. Goya, Y. Ge, A. C. Maiyar, and G. L. Firestone, "Characterization of sgk, a novel member of the serine/ threonine protein kinase gene family which is transcriptionally induced by glucocorticoids and serum," Molecular and Cellular Biology, vol. 13, no. 4, pp. 2031-2040, 1993.

[6] S. Waldegger, P. Barth, G. Raber, and F. Lang, "Cloning and characterization of a putative human serine/threonine protein kinase transcriptionally modified during anisotonic and isotonic alterations of cell volume," Proceedings of the National Academy of Sciences of the United States of America, vol. 94, no. 9, pp. 4440-4445, 1997.

[7] F. Lang, F. Artunc, and V. Vallon, "The physiological impact of the serum and glucocorticoid-inducible kinase SGK1," Current Opinion in Nephrology and Hypertension, vol. 18, no. 5, pp. 439448, 2009.

[8] C.-J. Cheng and C.-L. Huang, "Activation of PI3-kinase stimulates endocytosis of ROMK via Akt1/SGK1-dependent phosphorylation of WNK1," Journal of the American Society of Nephrology, vol. 22, no. 3, pp. 460-471, 2011.

[9] F. Lang, C. Stournaras, and I. Alesutan, "Regulation of transport across cell membranes by the serum- and glucocorticoidinducible kinase SGK1," Molecular Membrane Biology, vol. 31, no. 1, pp. 29-36, 2014.

[10] C. M. Taniguchi, B. Emanuelli, and C. R. Kahn, "Critical nodes in signalling pathways: insights into insulin action," Nature Reviews Molecular Cell Biology, vol. 7, no. 2, pp. 85-96, 2006.

[11] M. M. Cohen Jr., "The AKT genes and their roles in various disorders," American Journal of Medical Genetics Part A, vol. 161, no. 12, pp. 2931-2937, 2013.

[12] M. Ishiki and A. Klip, "Minireview: recent developments in the regulation of glucose transporter- 4 traffic: new signals, locations, and partners," Endocrinology, vol. 146, no. 12, pp. 50715078, 2005.

[13] A. L. Albiston, H. R. Yeatman, V. Pham et al., "Distinct distribution of GLUT4 and insulin regulated aminopeptidase in the mouse kidney," Regulatory Peptides, vol. 166, no. 1-3, pp. 83-89, 2011.

[14] E. Gonzalez and T. E. McGraw, "Insulin signaling diverges into Akt-dependent and -independent signals to regulate the recruitment/docking and the fusion of GLUT4 vesicles to the plasma membrane," Molecular Biology of the Cell, vol. 17, no. 10, pp. 4484-4493, 2006.

[15] G. Seki, H. Yamada, S. Horita et al., "Physiological and pathophysiological roles of the electrogenic $\mathrm{Na}^{+}-\mathrm{HCO}_{3}^{-}$cotransporter NBCel," Open Journal of Molecular and Integrative Physiology, vol. 1, no. 2, pp. 9-16, 2011.

[16] M. Baum, "Insulin stimulates volume absorption in the rabbit proximal convoluted tubule," Journal of Clinical Investigation, vol. 79, no. 4, pp. 1104-1109, 1987.

[17] D. G. Fuster, I. A. Bobulescu, J. Zhang, J. Wade, and O. W. Moe, "Characterization of the regulation of renal $\mathrm{Na}^{+} / \mathrm{H}^{+}$exchanger NHE3 by insulin," The American Journal of Physiology-Renal Physiology, vol. 292, no. 2, pp. F577-F585, 2007.

[18] Y. Zheng, H. Yamada, K. Sakamoto et al., "Roles of insulin receptor substrates in insulin-induced stimulation of renal 
proximal bicarbonate absorption," Journal of the American Society of Nephrology, vol. 16, no. 8, pp. 2288-2295, 2005.

[19] M. Nakamura, O. Yamazaki, A. Shirai et al., "Preserved Na/ $\mathrm{HCO}_{3}$ cotransporter sensitivity to insulin may promote hypertension in metabolic syndrome," Kidney International, vol. 87, no. 3, pp. 535-542, 2015.

[20] D. S. Kempe, G. Siraskar, H. Fröhlich et al., "Regulation of renal tubular glucose reabsorption by Akt2/PKB $\beta$," American Journal of Physiology-Renal Physiology, vol. 298, no. 5, pp. F1113-F1117, 2010.

[21] D. S. Kempe, T. F. Ackermann, K. M. Boini et al., "Akt2/ PKBbeta-sensitive regulation of renal phosphate transport," Acta Physiologica (Oxford), vol. 200, no. 1, pp. 75-85, 2010.

[22] A. D. Moes, N. van der Lubbe, R. Zietse, J. Loffing, and E. J. Hoorn, "The sodium chloride cotransporter SLC12A3: new roles in sodium, potassium, and blood pressure regulation," Pflugers Archiv European Journal of Physiology, vol. 466, no. 1, pp. 107-118, 2014.

[23] F. H. Wilson, S. Disse-Nicodème, K. A. Choate et al., "Human hypertension caused by mutations in WNK kinases," Science, vol. 293, no. 5532, pp. 1107-1112, 2001.

[24] J. A. McCormick and D. H. Ellison, "The WNKs: atypical protein kinases with pleiotropic actions," Physiological Reviews, vol. 91, no. 1, pp. 177-219, 2011.

[25] E. J. Hoorn and D. H. Ellison, "WNK kinases and the kidney," Experimental Cell Research, vol. 318, no. 9, pp. 1020-1026, 2012.

[26] S. Uchida, "Pathophysiological roles of WNK kinases in the kidney," Pflugers Archiv, vol. 460, no. 4, pp. 695-702, 2010.

[27] D. Takahashi, T. Mori, N. Nomura et al., "WNK4 is the major WNK positively regulating NCC in the mouse kidney," Bioscience Reports, vol. 34, no. 3, pp. 195-205, 2014.

[28] M. Wakabayashi, T. Mori, K. Isobe et al., "Impaired KLHL3mediated ubiquitination of WNK4 causes human hypertension," Cell Reports, vol. 3, no. 3, pp. 858-868, 2013.

[29] H. Nishida, E. Sohara, N. Nomura et al., "Phosphatidylinositol 3-kinase/akt signaling pathway activates the WNK-OSR1/ SPAK-NCC phosphorylation cascade in hyperinsulinemic db/db mice," Hypertension, vol. 60, no. 4, pp. 981-990, 2012.

[30] R. Komers, S. Rogers, T. Oyamat et al., "Enhanced phosphorylation of $\mathrm{Na}^{+}-\mathrm{Cl}^{-}$co-transporter in experimental metabolic syndrome: role of insulin," Clinical Science, vol. 123, no. 11, pp. 635-647, 2012.

[31] F. Lang, C. Böhmer, M. Palmada, G. Seebohm, N. StrutzSeebohm, and V. Vallon, "(Patho)physiological significance of the serum- and glucocorticoid-inducible kinase isoforms," Physiological Reviews, vol. 86, no. 4, pp. 1151-1178, 2006.

[32] V. A. Stevens, S. Saad, P. Poronnik, C. A. Fenton-Lee, T. S. Polhill, and C. A. Pollock, "The role of SGK-1 in angiotensin II-mediated sodium reabsorption in human proximal tubular cells," Nephrology Dialysis Transplantation, vol. 23, no. 6, pp. 1834-1843, 2008.

[33] F. Lang and E. Shumilina, "Regulation of ion channels by the serum- and glucocorticoid-inducible kinase SGK1," The FASEB Journal, vol. 27, no. 1, pp. 3-12, 2013.

[34] A. C. Pao, "SGK regulation of renal sodium transport," Current Opinion in Nephrology and Hypertension, vol. 21, no. 5, pp. 534540, 2012.

[35] N. Faresse, D. Lagnaz, A. Debonneville et al., "Inducible kidneyspecific Sgk1 knockout mice show a salt-losing phenotype," American Journal of Physiology: Renal Physiology, vol. 302, no. 8, pp. F977-F985, 2012.
[36] D. J. Rozansky, T. Cornwall, A. R. Subramanya et al., "Aldosterone mediates activation of the thiazide-sensitive $\mathrm{Na}-\mathrm{Cl}$ cotransporter through an SGK1 and WNK4 signaling pathway," The Journal of Clinical Investigation, vol. 119, no. 9, pp. 2601-2612, 2009.

[37] V. Vallon, J. Schroth, F. Lang, D. Kuhl, and S. Uchida, "Expression and phosphorylation of the $\mathrm{Na}^{+}-\mathrm{Cl}^{-}$cotransporter NCC in vivo is regulated by dietary salt, potassium, and SGK1," American Journal of Physiology-Renal Physiology, vol. 297, no. 3, pp. F704-F712, 2009.

[38] C. Ronzaud, D. Loffing-Cueni, P. Hausel et al., "Renal tubular NEDD4-2 defciency causes NCC-mediated salt-dependent hypertension," Journal of Clinical Investigation, vol. 123, no. 2, pp. 657-665, 2013.

[39] A. M. Ring, Q. Leng, J. Rinehart et al., "An SGK1 site in WNK4 regulates $\mathrm{Na}^{+}$channel and $\mathrm{K}^{+}$channel activity and has implications for aldosterone signaling and $\mathrm{K}^{+}$homeostasis," Proceedings of the National Academy of Sciences of the United States of America, vol. 104, no. 10, pp. 4025-4029, 2007.

[40] S. Saad, D. J. Agapiou, X.-M. Chen, V. Stevens, and C. A. Pollock, "The role of Sgk-1 in the upregulation of transport proteins by PPAR- $\gamma$ agonists in human proximal tubule cells," Nephrology Dialysis Transplantation, vol. 24, no. 4, pp. 1130-1141, 2009.

[41] J. Geibel, G. Giebisch, and W. F. Boron, "Angiotensin II stimulates both $\mathrm{Na}^{+}-\mathrm{H}^{+}$exchange and $\mathrm{Na}^{+} / \mathrm{HCO}^{-}$cotransport in the rabbit proximal tubule," Proceedings of the National Academy of Sciences of the United States of America, vol. 87, no. 20, pp. 7917-7920, 1990.

[42] C. Boehmer, H. M. Embark, A. Bauer et al., "Stimulation of renal $\mathrm{Na}^{+}$dicarboxylate cotransporter 1 by $\mathrm{Na}^{+} / \mathrm{H}^{+}$exchanger regulating factor 2 , serum and glucocorticoid inducible kinase isoforms, and protein kinase B," Biochemical and Biophysical Research Communications, vol. 313, no. 4, pp. 998-1003, 2004.

[43] K. Ho, C. G. Nichols, W. J. Lederer et al., "Cloning and expression of an inwardly rectifying ATP-regulated potassium channel," Nature, vol. 362, no. 6415, pp. 31-38, 1993.

[44] A. R. Rodan and C.-L. Huang, "Distal potassium handling based on flow modulation of maxi-K channel activity," Current Opinion in Nephrology and Hypertension, vol. 18, no. 4, pp. 350355, 2009.

[45] C. W. Lindsley, "The Akt/PKB family of protein kinases: a review of small molecule inhibitors and progress towards target validation: a 2009 update," Current Topics in Medicinal Chemistry, vol. 10, no. 4, pp. 458-477, 2010.

[46] A. Bellacosa, C. C. Kumar, A. D. Cristofano, and J. R. Testa, "Activation of AKT kinases in cancer: implications for therapeutic targeting," Advances in Cancer Research, vol. 94, no. 1, pp. 29-86, 2005.

[47] C. S. Mitsiades, N. Mitsiades, and M. Koutsilieris, "The Akt pathway: molecular targets for anti-cancer drug development," Current Cancer Drug Targets, vol. 4, no. 3, pp. 235-256, 2004.

[48] T. W. Miller, B. N. Rexer, J. T. Garrett, and C. L. Arteaga, "Mutations in the phosphatidylinositol 3-kinase pathway: role in tumor progression and therapeutic implications in breast cancer," Breast Cancer Research, vol. 13, no. 6, article 224, 2011.

[49] S. Chia, S. Gandhi, A. A. Joy et al., "Novel agents and associated toxicities of inhibitors of the PI3k/Akt/mtor pathway for the treatment of breast cancer," Current Oncology, vol. 22, no. 1, pp. 33-48, 2015.

[50] Y. Oki, M. Fanale, J. Romaguera et al., "Phase II study of an AKT inhibitor MK2206 in patients with relapsed or refractory lymphoma," British Journal of Haematology, 2015. 
[51] F. Lang, N. Perrotti, and C. Stournaras, "Colorectal carcinoma cells-regulation of survival and growth by SGK1," International Journal of Biochemistry and Cell Biology, vol. 42, no. 10, pp. 15711575, 2010.

[52] J. W. Yoon, R. Gilbertson, S. Iannaccone, P. Iannaccone, and D. Walterhouse, "Defining a role for Sonic hedgehog pathway activation in desmoplastic medulloblastoma by identifying GLI1 target genes," International Journal of Cancer, vol. 124, no. 1, pp. 109-119, 2009.

[53] O. Nasir, K. Wang, M. Föller et al., "Relative resistance of SGK1 knockout mice against chemical carcinogenesis," IUBMB Life, vol. 61, no. 7, pp. 768-776, 2009.

[54] S. T. Towhid, G.-L. Liu, T. F. Ackermann et al., "Inhibition of colonic tumor growth by the selective SGK inhibitor EMD638683," Cellular Physiology and Biochemistry, vol. 32, no. 4, pp. 838-848, 2013.

[55] A. B. Sherk, D. E. Frigo, C. G. Schnackenberg et al., "Development of a small-molecule serum- and glucocorticoid-regulated kinase- 1 antagonist and its evaluation as a prostate cancer therapeutic," Cancer Research, vol. 68, no. 18, pp. 7475-7483, 2008. 


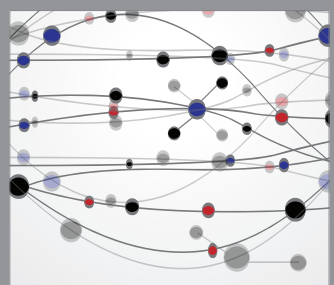

The Scientific World Journal
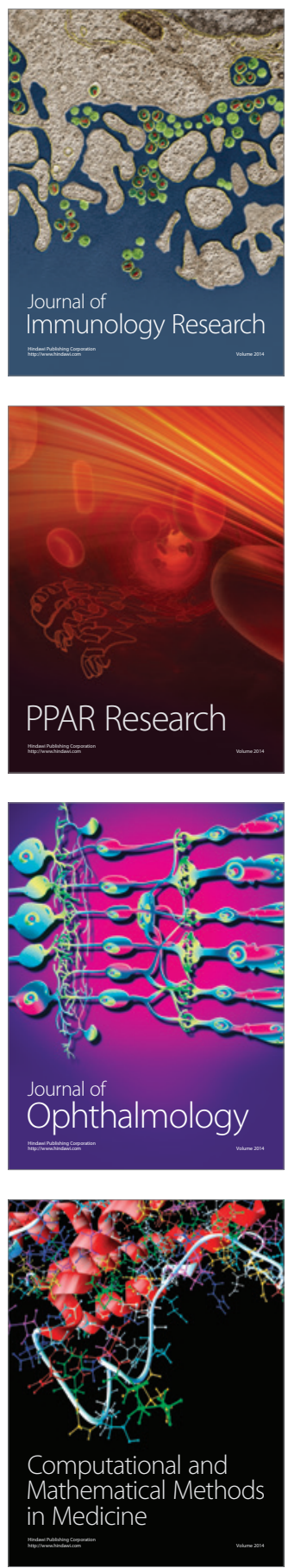

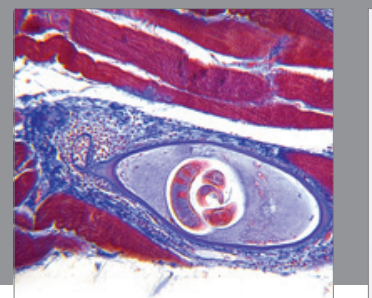

Gastroenterology

Research and Practice
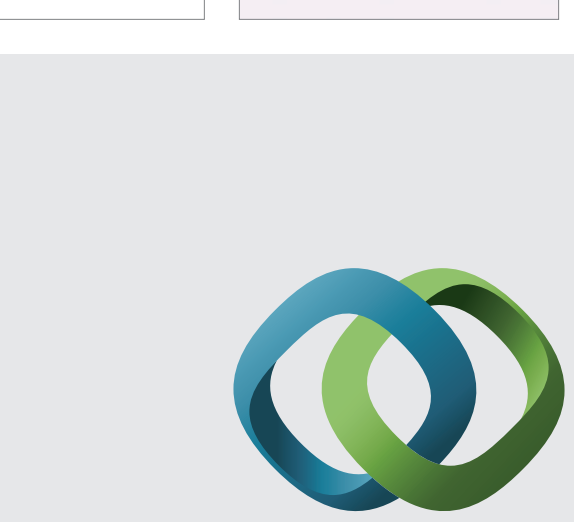

\section{Hindawi}

Submit your manuscripts at

http://www.hindawi.com
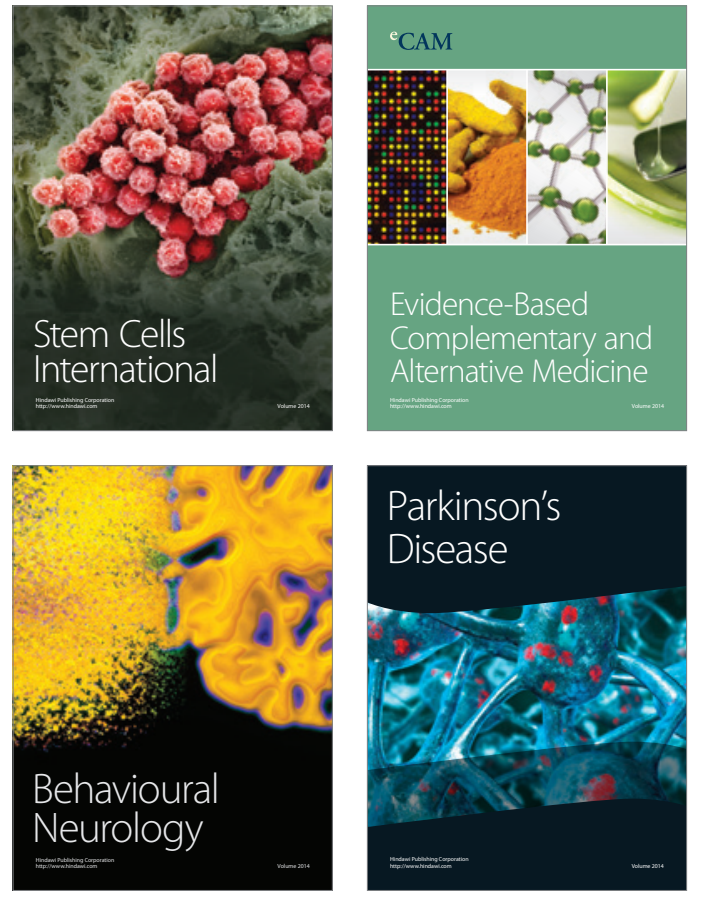
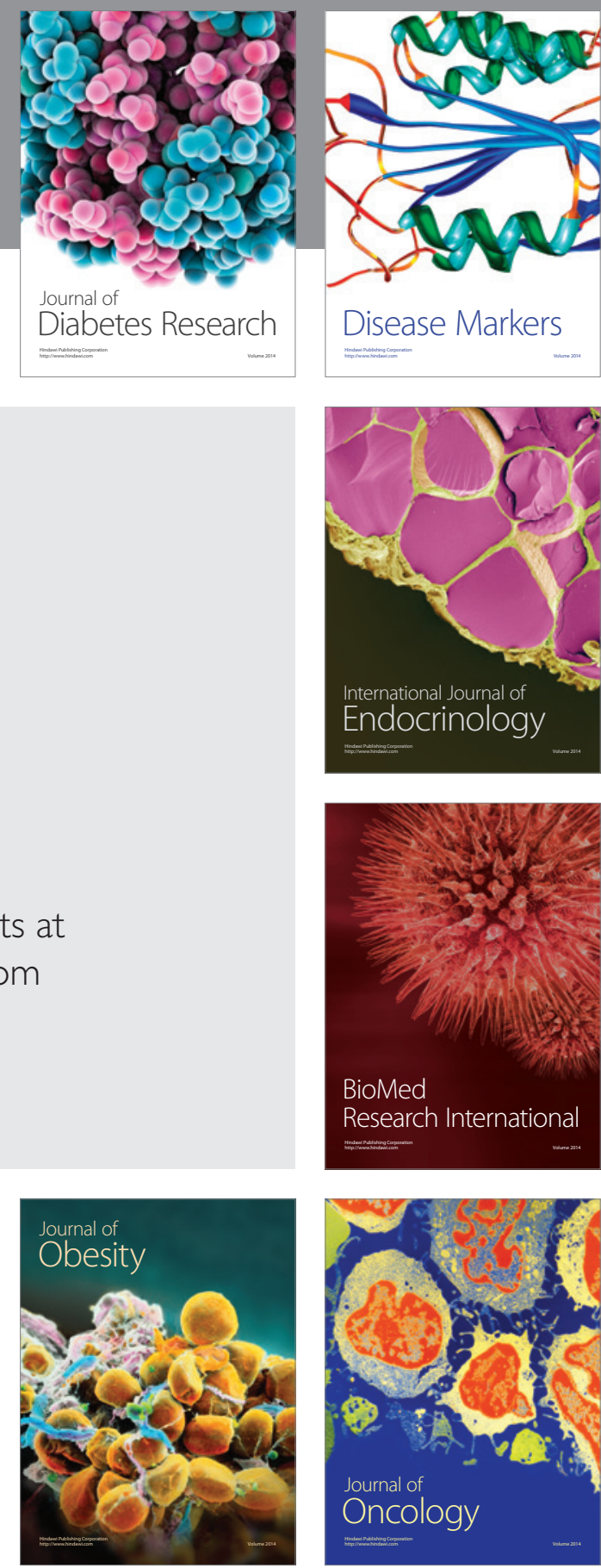

Disease Markers
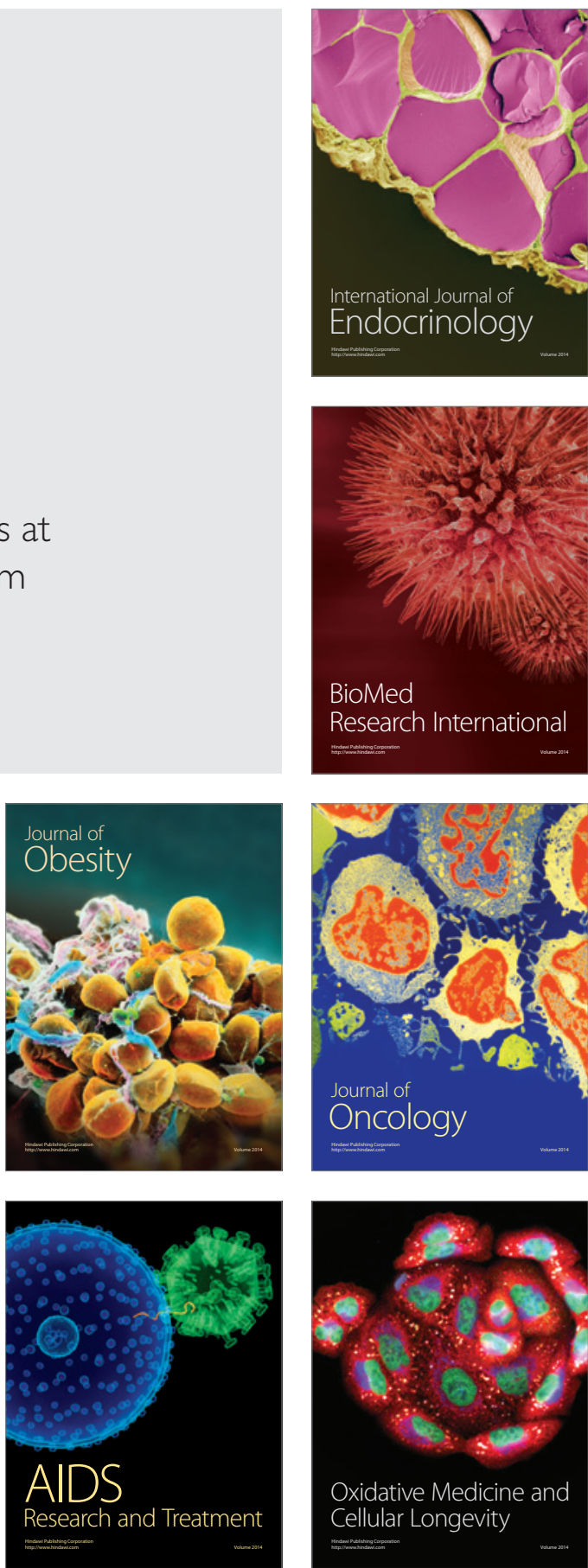